\title{
Un Modelo Computacional de la Transmisión del Dengue por Autómatas Celulares.
}

\section{A Computational Model of Dengue Transmission by Cellular Automata.}

\author{
Neisser Pino Romero * (iD and Gabriel Wainer ${ }^{\dagger}$ (iD
}

Received, Set. 24, 2019

Accepted, Nov. 22, 2019

How to cite this article:

Pino, N., Wainer, G. Un Modelo Computacional de la Transmisión del Dengue por Autómatas Celulares. Selecciones Matemáticas. 2019; 6(2):217-224. http://dx.doi.org/10.17268/sel .mat.2019.02.08

\section{Resumen}

El trabajo de investigación presenta un modelo computacional por Autómatas Celulares (Cell-DEVS) aplicado a la transmisión de la enfermedad Dengue, propagada por mosquitos hacia una población susceptible. Este modelo tendrá un enfoque de multinivel para poder considerar interacciones exógenas como temperatura. Esto permitirá poder visualizar los puntos críticos donde la reproducción de mosquitos es mucho mayor, y así realizar estrategias de control.

Palabras clave. Epidemiología matemática. Autómatas celulares, Formalismo DEVS.

\begin{abstract}
The research paper presents a computational model by Cellular Automata (Cell-DEVS) applied to the transmission of Dengue disease, spread by mosquitoes to a susceptible population. This model will have a multilevel approach to consider exogenous interactions such as temperature. This will allow to be able to visualize the critical points where the mosquito reproduction is much greater, and thus carry out control strategies.
\end{abstract}

Keywords: Mathematical epidemiology. Cellular automata. DEVS formalism.

1. Introducción. El dengue es una de las Enfermedades re-emergentes de mayor importancia en la región de las Américas y en el Perú, expone a más del $50 \%$ de la población peruana al riesgo de desarrollar la enfermedad por el espacio peruano infestado por Aedes aegypti como lo determina la Dirección General de Epidemiología. El comportamiento biológico del vector Aedes Aegypti depende de varios factores climatológicos y topográficos, donde el análisis del suelo siempre se tiene en cuenta: la temperatura, la precipitación y la altura. También se analiza la población existente del vector en base a modelos planteados en otros países como pueden ser Brasil, Colombia, Ecuador y Bolivia. Por lo cual, la matemática como una ciencia a servicio de la humanidad ha propuesto diferentes modelos para describir su comportamiento en la interacción del hombre y de los mosquitos. De forma similar, la computación ha brindado importantes herramientas informáticas para graficar y así poder visualizar la dinámica entre la población humana y de los mosquitos y cómo se puede realizar intervenciones epidemiológicas para prevenir y controlar epidemias que transmiten estos mosquitos (Dengue, Zika y Chikungunya). [2, 3, 4, 6, 10, 11].

2. Modelo Matemático del Dengue. La matemática mediante la generación de modelos aplicados a la Epidemiología ha brindado una variedad de herramientas de tal forma que se pueda describir, estudiar,

\footnotetext{
* Facultad de Ciencias y Filosofía, Universidad Peruana Cayetano Heredia. Universidad Nacional Mayor de San Marcos. Lima, Perú. (neisser.pino@upch.pe), (neisser@unmsm.edu.pe)

${ }^{\dagger}$ Carleton University, Department of Systems and Computer Engineering. Ottawa, Canadá. (gwainer@sce. carleton . ca).
} 
analizar e inferir conclusiones a partir de un sistema de ecuaciones. Se puede recurrir a una extensa bibliografía donde se propone el modelo y se realiza todo el estudio matemático de la existencia, unicidad de soluciones, la positividad de las soluciones, y de forma más detallada el estudio de la estabilidad (local o global) del modelo para el estado estacionario del modelo según la Teoría cualitativa de las soluciones de un Sistema de Ecuaciones Diferenciales Ordinarias. [2, 3, 4, 6, 7, 8].

Entre los estudios realizados se ha escogido algunos de diferentes países que afrontan esta problemática de la transmisión de enfermedades por mosquitos, de forma particular el Dengue. El trabajo de María Kuno donde realiza un estudio detallado para Bolivia [6], el trabajo de Sepúlveda-Salcedo [13] y Medina [10] para el caso de Colombia y para el caso del Perú se tiene el trabajo de Roxana López [7] y César Cabezas [1]. Todos excelentes trabajos sobre el Dengue desde de un punto de vista matemático y epidemiológico. Sus trabajos contienen demostraciones matemáticos que garantizan la estabilidad local y son complementados con simulaciones computacionales e interpretaciones epidemiológicas que brindan nuevos horizontes de intervención de prevención y control por parte de los Ministerios de Salud. [2, 3, 4, 5, 14, 19].

3. Modelo Computacional del Dengue. Los Autómatas Celulares pueden expresar un modelo matemático para un sistema dinámico, conformado por un conjunto de celdas o células que adquieran distintos estados o valores. Estos estados son alterados de un instante a otro en unidades de tiempo discreto, es decir, que se puede cuantificar con valores enteros a intervalos regulares. De esta manera, este conjunto de células logran una evolución según una determinada expresión matemática, que es sensible a los estados de las células vecinas, la cual se le denomina como reglas de transición local. Según Wainer y Giambiasi en [17], el formalismo Cell-DEVS fue presentado como la combinación de los autómatas celulares (A.C.) y el formalismo DEVS, cada célula está definida como un modelo atómico, y se determina el procedimiento para acoplar las células, es decir, se define la interacción de las células entre sí.[12, 16, 18].

El modelo computacional mediante autómatas celulares se fundamenta en la definición de las reglas correspondientes para poder generar la simulación computacional desde la perspectiva de los autómatas celulares mediante el formalismo DEVS. El modelamiento computacional por Autómatas Celulares (CellDEVS) se expresará como un sistema de multi-nivel, es decir, se definirá diferentes células en diferentes niveles de tal forma que se puede definir reglas para cada nivel pero que se relacionan entre sí. Con esta definición se puede conectar interacciones entre las poblaciones humanas y la de los mosquitos. De forma gráfica, la construcción del modelo sería de la siguiente forma. [5, 9, 12, 19].

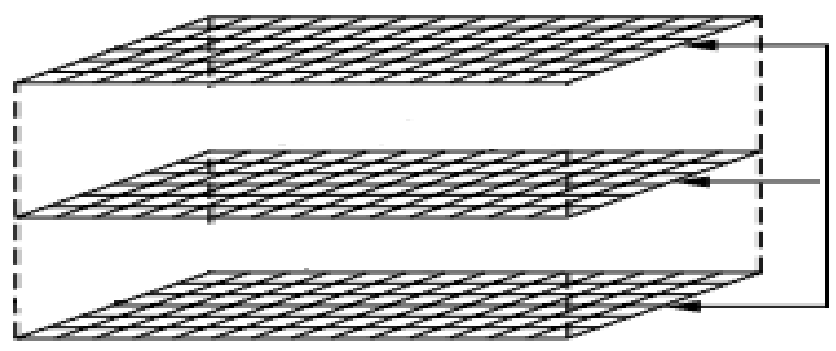

FIgura 3.1. Modelo Computacional con Tres Niveles de Construcción. Fuente: Discrete-Event Modeling and Simulation, Wainer (2009)

Se puede definir tantos niveles como se requiera. Este detalle en el modelamiento se puede aprovechar para lograr añadir consideraciones externas que afecten la superficie principal. Y en cada nivel se puede definir un vecindario diferente si se desea. Por lo cual, la definición del vecindario puede variar de acuerdo a la dinámica de la célula que puede interactuar con las demás. [15, 17, 18].

3.1. Reglas del Modelo Computacional. Las reglas que se definirán para representar la dinámica de transición de los diferentes estados de las células (personas y mosquitos) de forma principal del modelo. Pero también se puede considerar la temperatura que pueda estar presente debido que la temperatura ayuda a una mayor reproducción de los mosquitos. Como se puede observar, en la construcción de un modelo matemático se define hipótesis para la adecuada formulación del mismo. Por ende, hay una similitud en la construcción de un modelo matemático y un modelo computacional. Por consiguiente, las hipótesis del modelo del Dengue, de forma sencilla, serían los siguientes: [3, 6, 10, 11, 15, 14]. 

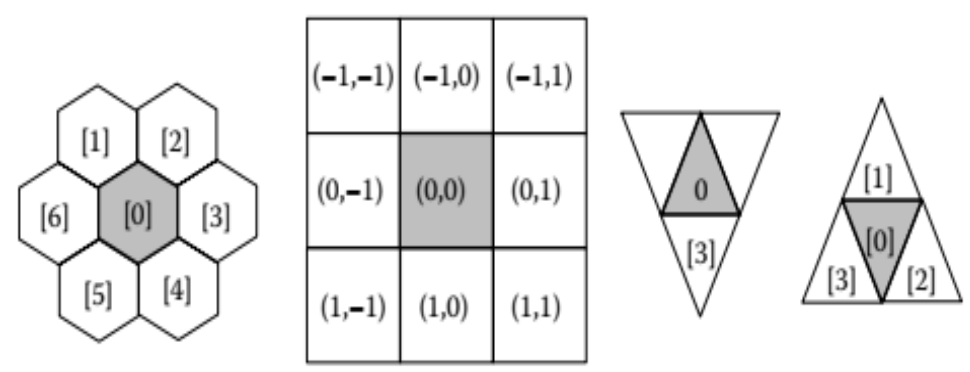

FIGURA 3.2. Definición de diferentes tipos de Vecindarios del Modelo Computacional. Fuente: Discrete-Event Modeling and Simulation, Wainer (2009)

- La reproducción de las Personas se da en ausencia de los mosquitos.

- El contagio de un Humano Susceptible se da cuando interactúa con un mosquito infectado.

- El Humano infectado se llega a recuperar con la atención de los susceptibles y los recuperados.

- El tiempo de vida promedio de un Humano Susceptible es mayor que un Humano Infectado.

- La reproducción de los mosquitos se da en la presencia de otros mosquitos. La temperatura ayuda a una reproducción más rápida.

- Un mosquito susceptible contrae la enfermedad mediante el contacto de un Humano Infectado.

- Los mosquitos mueren por la intervención de los Humanos Susceptibles y Recuperados, o también por finalizar su tiempo de vida promedio.

En primer lugar, definiremos el número correspondiente a cada variable de nuestro modelo computacional. De tal forma, será más sencillo conocer la interacción de las poblaciones. [9, 12, 17].

$$
\text { Poblaciones }=\left\{\begin{array}{lll}
0 & : & \text { Celda Vacía (Blanco) } \\
1 & : & \text { Celda de Persona Susceptible (Verde) } \\
2 & : & \text { Celda de Persona Infectada (Rojo) } \\
3 & : & \text { Celda de Persona Recuperada (Azul) } \\
8 & : & \text { Celda de Mosquito Susceptible (Amarillo) } \\
9 & : & \text { Celda de Mosquito Infectado (Magenta) }
\end{array}\right.
$$

Habiendo definido el número correspondiente a las células, procederemos a realizar la tabla de reglas. Un detalle importante va ser la consideración del clima donde se ubique los mosquitos. Pues esto acelera enormemente su reproducción. [9, 12, 15, 16, 18].

$$
\text { Temperatura }=\left\{\begin{array}{lll}
12-16 & : & \text { Temperatura Fría (Plomo) } \\
17-22 & : & \text { Temperatura Templada (Naranja Claro) } \\
23-30 & : & \text { Temperatura Calurosa (Naranja) }
\end{array}\right.
$$

Con estas definiciones, se podrá formalizar las Reglas de Transición de nuestro modelo computacional.

Después de haber definido la reglas de transición en el cuadro (3.1) que contiene 12 reglas en total se puede pasar a realizar la simulación. El mayor detalle de este cuadro tiene que ver con las reglas $\mathbf{7 , 8}$, 9 debido que contiene la reproducción de los mosquitos que dependen de la Temperatura que hay en ese momento. La consideración de la variable del clima en un modelo humano-vector es importante porque se enfoca en los puntos críticos de la ubicación de los mosquitos. [1, 4, 6, 12, 15, 18].

3.2. Simulación Computacional. Como toda simulación computacional que proviene de un modelo matemático, se debe definir los parámetros y las poblaciones iniciales. De forma similar definiremos las poblaciones iniciales y también los parámetros correspondientes al clima para poder analizar que las variantes de temperatura en distintas zonas puede afectar enormemente en la propagación poblacional de los mosquitos. Se considerará una malla cuadrangular de $20 \times 20$ (400 células) que se ubicarán dentro de todo el territorio considerado para la simulación computacional mediante Autómatas Celulares. [9, 12, 15, 18]. 


\begin{tabular}{|c|c|l|c|}
\hline Regla & Estado Inicial & \multicolumn{1}{|c|}{ Regla de Transición } & Estado Final \\
\hline 1 & 0 & $(\#(1)+\#(3)) \geq 1 \wedge(\#(8)+\#(9))=0 \wedge P b\left(\beta_{1}\right)$ & 1 \\
\hline 2 & 1 & $\#(2) \geq 1 \wedge P b\left(\beta_{2}\right)$ & 2 \\
\hline 3 & 1 & Tiempo de Vida Susceptible Humano & 0 \\
\hline 4 & 2 & $(\#(1)+\#(3)) \geq 1 \wedge P b\left(\phi_{1}\right) \wedge t_{\text {Recuperacion }}$ & 3 \\
\hline 5 & 2 & Tiempo de Vida Infectado Humano & 0 \\
\hline 6 & 3 & Tiempo de Inmunidad Humano & 1 \\
\hline 7 & 0 & {$\left[(\#(8)+\#(9)) \geq 3 \wedge 12<\right.$ Temp. $\left.<16 \wedge P b\left(\phi_{2}\right)\right]$} & 8 \\
\hline 8 & 0 & {$\left[(\#(8)+\#(9)) \geq 2 \wedge 17<\right.$ Temp. $\left.<22 \wedge P b\left(\phi_{2}\right)\right]$} & 8 \\
\hline 9 & 0 & {$\left[(\#(8)+\#(9)) \geq 1 \wedge 23<\right.$ Temp. $\left.<30 \wedge P b\left(\phi_{2}\right)\right]$} & 8 \\
\hline 8 & 8 & $\#(2) \geq 1 \wedge P b\left(\phi_{3}\right)$ & 9 \\
\hline 9 & 8 & $(\#(1)+\#(3)) \geq 2 \wedge P b\left(\phi_{4}\right)$ & 0 \\
\hline 10 & 8 & Tiempo de Vida Mosquito & 0 \\
\hline 11 & 9 & $(\#(1)+\#(3)) \geq 2 \wedge P b\left(\phi_{4}\right)$ & 0 \\
\hline 12 & 9 & Tiempo de Vida Mosquito & \\
\hline
\end{tabular}

\begin{tabular}{|c|c|}
\hline Población Susceptible Humano & $S(0)=183$ \\
\hline Población Infectada Humana & $I(0)=36$ \\
\hline Población Recuperada Humana & $R(0)=18$ \\
\hline Población Susceptible Mosquito & $M_{S}(0)=34$ \\
\hline Población Infectada Mosquito & $M_{I}(0)=44$ \\
\hline
\end{tabular}

Se presenta la posición inicial de las poblaciones en la malla donde la ubicación donde se comience la simulación determinará el futuro de la propagación en tiempo y espacio. La interacción de cada célula depende de su vecindario y de la condiciones determinadas por las reglas del modelo expresadas en el cuadro (3.1) con lo cual se irá propagando la enfermedad. Un detalle que no se ha formalizado en la construcción del modelo computacional es su estructura dentro del formalismo DEVS, esta estructura se puede construir como se indica en el libro de Wainer [18], esto es porque nos enfocaremos en su transición computacional y su proceso evolutivo para cada población. El software que se utilizará es el CD++ debido que usa el formalismo DEVS con una interfaz dinámica que permite la programación de forma sencilla. [9, 12, 17, 19].

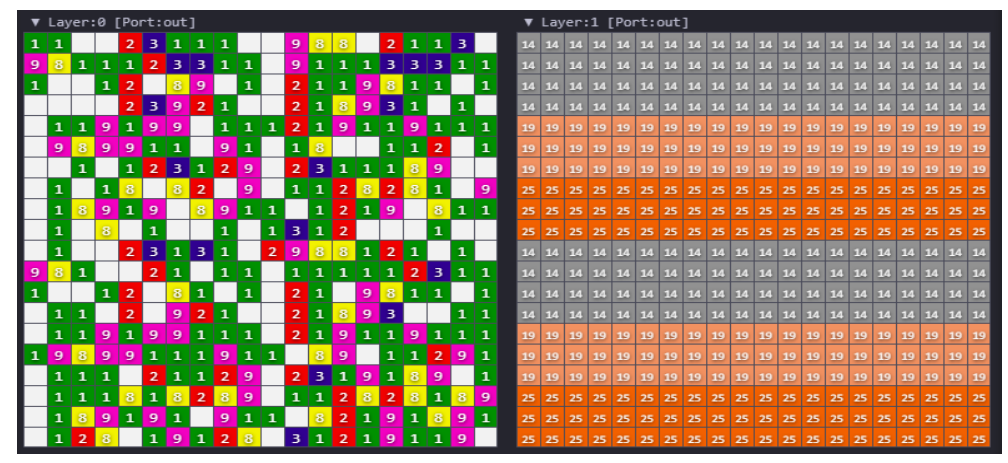

FIgURA 3.3. Estado Inicial de la Simulación del Modelo Computacional

La gráfica del estado inicial, se analiza dos cuadros para su respectiva interpretación, el lado izquierdo contiene la malla de la Superficie y el lado derecho contiene la malla del Clima. A partir de este estado inicial, se podrá realizar la evolución de la interacción entre las poblaciones de las Personas y de los mosquitos. La malla del clima se ha inicializado de forma uniforme pero en el tiempo variará de acuerdo a un crecimiento aleatorio debido que la temperatura no aumenta o disminuye de forma uniforme. Con estas consideraciones, procederemos a realizar la simulación computacional del modelo. Se ha considerado un 
tiempo de compilación de 400 segundos (40 estados). Esto nos ayudará a analizar como interactúan las poblaciones del modelo y también como va cambiando en el tiempo la temperatura. [1, 3, 12, 15, 18$]$.

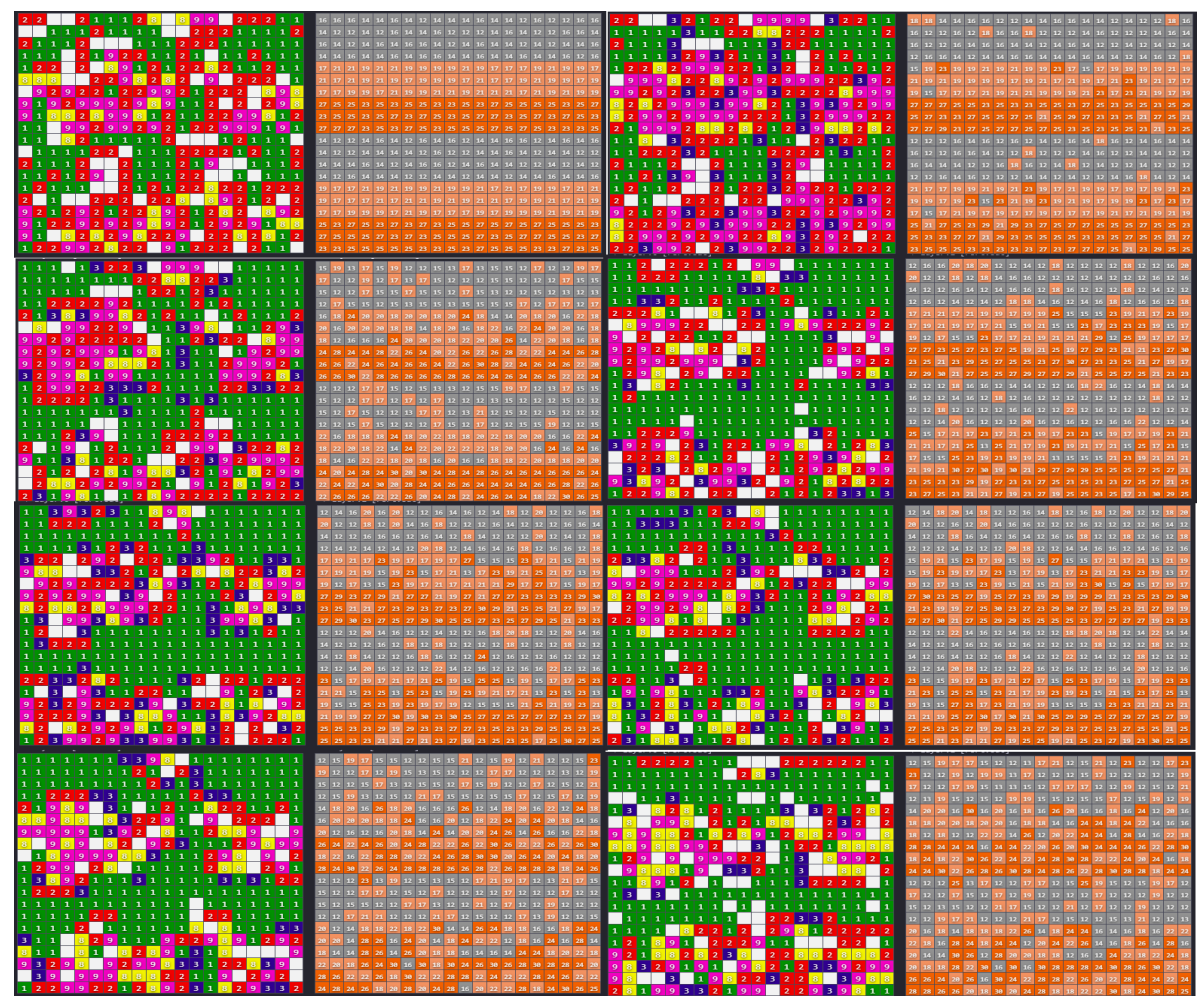

FIGURA 3.4. Evolución en el Tiempo-Espacio del Modelo Computacional del Dengue

Después de obtener la simulación en el tiempo de compilación, se puede analizar de forma epidemiológica y computacional la evolución del modelo. No se ploteará en el tiempo la transición de la temperatura debido nos centraremos en las poblaciones. [4, 12, 15, 18].

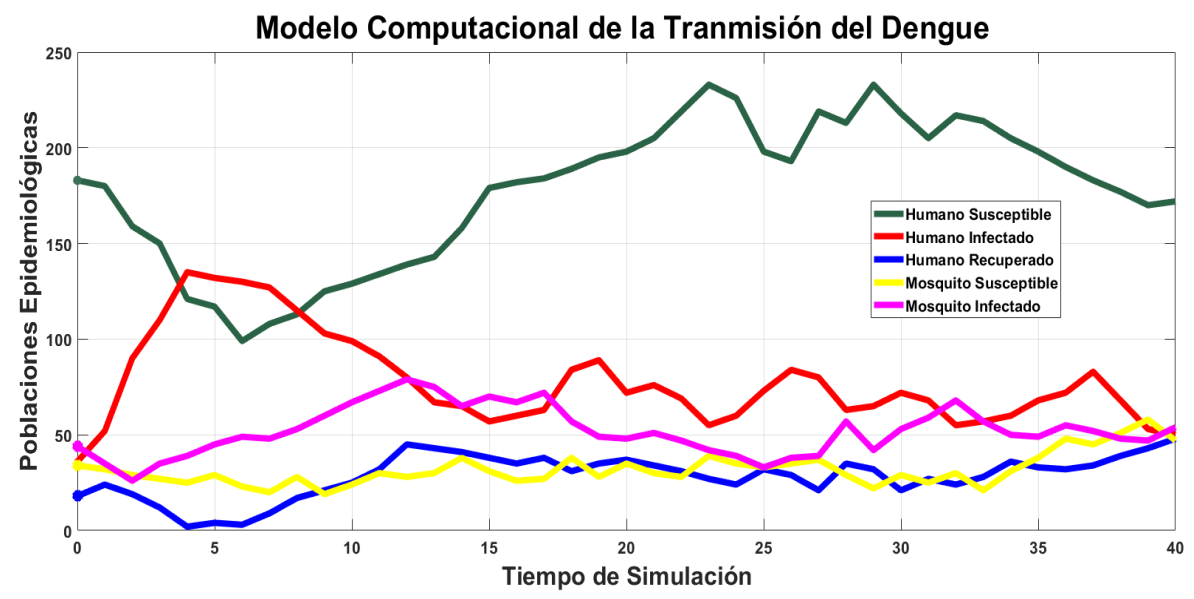

3.3. Interpretación del Modelo Computacional. La construcción del modelo computacional mediante reglas sencillas ha permitido la generación de la gráfica anterior donde se puede analizar a través del tiempo como va evolucionando cada población, tanto de los humanos como de los mosquitos. Es verdad que se ha considerado una pequeña malla rectangular para analizar la situación de contacto que tienen los humanos con los mosquitos tanto sea para el contagio (mosquito a humano como de humano a mosquito) como para la mortalidad (natural o por enfermedad (para el humano) o por estrategia de control (exterminación de mosquitos)). Hay muchos puntos a considerar en este modelo que faltaría incluir para poder aproximarlo 
más a la realidad que se suscita en las regiones que afrontan esta problemática. Pero dentro de sus limitaciones, el modelo representa muy bien la dinámica de interacción entre el humano y los mosquitos, y como también afecta la temperatura en la reproducción de los mosquitos. [1, 3, 6, 14, 19].

La población susceptible siempre se ha mantenido por encima de todas las poblaciones por mientras que la infectada tiene un umbral epidémico par luego descender. La población de los mosquitos tienen un crecimiento leve pero se mantienen en una población constante, esto es debido a la temperatura que que genera una reproducción rápida en ciertas zonas, con lo cual se mantiene la población de mosquitos susceptibles. Por otro lado, la vida promedio del mosquito es bastante corta (10 a 30 días); pero sin lugar a duda su ciclo de vida es muy activa donde su territorio de expansión es pequeño en cuanto a su traslado pero no a su propagación. [2, 3, 6, 8, 10, 11].

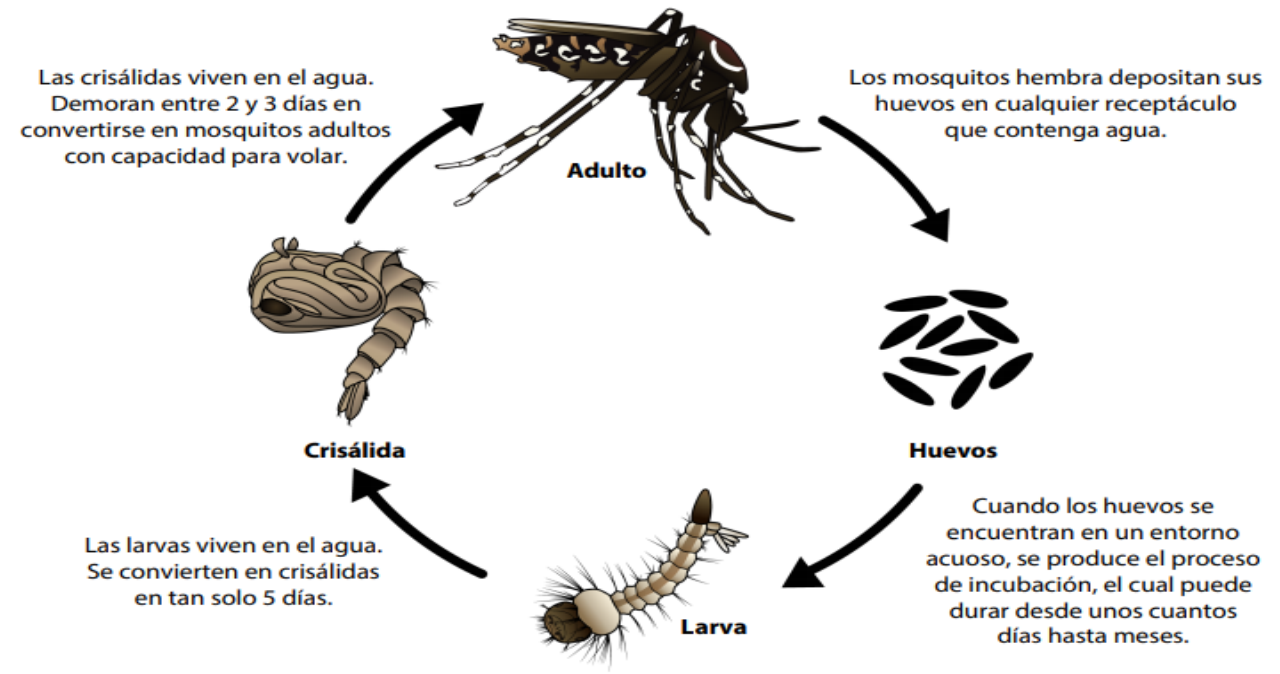

FIGURA 3.5. Fuente: Ciclo de vida del mosquito (Aedes aegypti), C.D.C. (2018)

Finalmente, el modelo computacional brinda mucha información para analizar como se puede prevenir y control pequeñas áreas de mosquitos donde la temperatura puede acelerar el proceso de reproducción. Las poblaciones de humanos siempre estarán presentes para contrarrestar el crecimiento de los mosquitos. Pero solamente basta una pequeñisima parte de la población que se contagie de la enfermedad para generar una epidemia que puede costar vidas a la sociedad. Por otro lado, también se podría añadir más niveles que modelen más variables que puedan generar una reproducción más rápida de los mosquitos como los lugares con aguas descubiertas entre otras. Esto ayudaría a mejorar el modelo computacional. [5, 14, 15, 18, 17$]$.

4. Discusiones. Después de haber realizado la simulación computacional y las intepretaciones epidemiológicas respectivas a la interacción que existe entre las personas y los mosquitos, y además que el mosquito tiene una relación directa con el clima debido que incrementa la velocidad de reproducción. El modelo computacional ha sido dividido en dos niveles: superficie (humanos y mosquitos) y clima (temperatura). Esto permite ver la dinámica de la temperatura en cuanto a su relación con el mosquito, pero también se puede añadir más niveles donde se puedan considerarse más variables que intervengan en la dinámica de la propagación de la enfermedad, en nuestro caso el Dengue o también como podría ser el Zika o la Chikungunya. $[3,6,10,11,13]$.

Desde el punto de vista computacional, esto conlleva un mayor costo computacional (mayor cantidad de operaciones y de estructuras) y esto se refleja en el tamaño de la malla. También el formalismo lógico debe ser lo más preciso posible debido que los niveles aunque estén separados se mantienen unidos porque interactúan entre sí. En este contexto, el formalismo DEVS permite una mejor estructura de construcción de la simulación computacional (CD++). [9, 15, 16, 18].

5. Conclusiones. Se ha desarrollado de manera puntual un modelo computacional por Autómatas Celulares desde el formalismo DEVS para describir la dinámica de transmisión del Dengue. La interacción entre las personas y los mosquitos siempre ha habido en las zonas tropicales, debido que este tipo de climas es muy provechoso para los mosquitos. [15, 18, 19]. 
Hay diversos modelos matemáticos que describen la dinámica de transmisión mediante las E.D.O. (el tiempo como variable independiente) y también como las E.D.P. (el tiempo y el espacio como variables independientes). Y la variable de la Temperatura en muchas ocasiones no se puede apreciar como afecta de manera directa al modelo. Por ende, en este modelo computacional con una construcción de dos niveles (superficie y clima) ayuda enormemente como la visualización y el análisis de la propagación poblacional de los mosquitos. [5, 6, 8, 10, 11, 15].

Por lo cual, se puede concluir lo siguiente:

- El modelamiento matemático aporta enormemente al estudio de la problemática de la transmisión del Dengue que afecta a diversos países de Sudamérica (Colombia, Brasil, Perú y Bolivia) según lo expuesto en la sección 2, y cómo han generado un impacto favorable a la sociedad de cada país.

- La Dirección General de Epidemiología (DGE) realiza estrategias de control y prevención, dentro de los lineamientos de la salud pública del Ministerio de Salud, para disminuir la cantidad de infectados por el Dengue debido que puede causar la muerte si no se atiende con prontitud.

- El modelamiento por autómatas celulares ayuda en la construcción de un modelo mediante reglas sencillas y lógicas sin tener una enorme formación en modelamiento matemático. Las reglas pueden incluir diferentes consideraciones que se traducirán en estados que cambian con el tiempo de simulación y el espacio del vecindario.

- El modelo computacional en Cell-DEVS por niveles permite una mejor visualización del impacto que genera la temperatura en la superficie para la reproducción de los mosquitos en el tiempo y espacio considerado.

ORCID and License

Neisser Pino Romero https://orcid.org/0000-0002-9865-5974,

Gabriel Wainer https://orcid.org/0000-0003-3366-9184.

This work is licensed under the Creative Commons Attribution-NoComercial-ShareAlike 4.0.

\section{Referencias}

[1] Cabezas S., C. Dengue en el Perú: Aportes para su diagnóstico y control. Rev. Perú. Med. Exp. Salud Pública, 2005; 22(3):212-228. Recuperado de http://www.scielo.org.pe/scielo.php?script=sci_arttext\&pid= S1726-46342005000300009

[2] Centro Nacional para Enfermedades Infecciosas Emergentes y Zoonóticas. Ciclo de vida del mosquito. División de Enfermedades Transmitidas por Vectores (Centers for Disease Control and Prevention) (2018). Recuperado de https: //www.cdc.gov/zika/pdfs/spanish/MosquitoLifecycle-sp.pdf

[3] Dirección General de Epidemiología. Situación Epidemiología del Dengue: Números de Casos. Centro Nacional de Epidemiología, Prevención y Control de Enfermedades, Ministerio de Salud (2018). Recuperado de http: / / www. dge • gob . pe/portal/docs/vigilancia/sala/2018/SE01/dengue.pdf

[4] Dirección General de Epidemiología. Sal de Situación, Perú SE 52-2018. Centro Nacional de Epidemiología, Prevención y Control de Enfermedades, Ministerio de Salud (2018). Recuperado de http://www. dge.gob.pe/portal/docs/ tools/teleconferencia/2019/SE012019/01.pdf

[5] Brauer, F.; Castillo-Chavez, C. Mathematical Models in Population Biology and Epidemiology. Springer US, 2001. Recuperado de https://link.springer.com/book/10.1007/978-1-4614-1686-9

[6] Kuno Fernández, M. Simulación de la Propagación del vector Aedes Aegypti, Transmisor de las Enfermedades: Dengue, Zika y Chikungunya en Bolivia. Tesis de Licenciatura, Facultad de Ciencias Puras y Naturales, Universidad Mayor de San Andrés, 2016. Recuperado de https://repositorio.umsa.bo/handle/123456789/10700

[7] López Cruz, R. A mathematical model for mosquito infestation. Selecciones Matemáticas, 2018; 6(1):14-18. Recuperado de http://revistas.unitru.edu.pe/index.php/SSMM/article/view/2438/2477

[8] López, L; Muñoz-Loaiza, A; Olivar-Tost, G; Betancourt, J. Modelo matemático para el control de la transmisión del Dengue. Rev. salud pública. 2012; 14(3):512-523. Recuperado de https://www.scielosp.org/article/ssm/ content/raw/?resource_ssm_path=/media/assets/rsap/v14n3/v14n3a14.pdf

[9] Bonaventura, M.; Wainer, G.; Castro, R. Graphical modeling and simulation of discrete-event systems with CD++ Builder. Simulation, 2013; 89(1):4-27. Recuperado de https:// journals.sagepub.com/doi/abs/10.1177/ 0037549711436267

[10] Medina Arce, Y; Ramos Tapia, J. Modelo matemático que explica mejor la afectación e identifica el patrón relevante en la difusión para el dengue en la zona urbana del municipio de Neiva. Entornos, 2017; 30(2):121-131. Recuperado de https: //dialnet.unirioja.es/descarga/articulo/6394863.pdf

[11] Mosquera, L; Perea, Milton. Modelo Matemático para la Enfermedad del Dengue. Boletín de Matemáticas, 2006; 13(2):176-185. Recuperado de https://revistas.unal.edu.co/index.php/bolma/article/view/ $40454 / 42298$

[12] Pino Romero, N.: López Cruz, R.; Wainer, G. Modelamiento computacional de la dinámica de transmisión sexual del VIH/SIDA mediante autómatas celulares (Cell-DEVS). Selecciones Matemáticas, 2018; 5(1):39-47. Recuperado de http://www . revistas.unitru.edu.pe/index.php/s.SMM/article/view/1986

[13] Sepúlveda-Salcedo, L; Vasilieva, O; Martínez-Romero, H; Arias-Castro, J. Ross McDonald: Un modelo para la dinámica del dengue en Cali, Colombia. Revista de Salud Pública, 2015; 17(5):749-761. Recuperado de https://revistas . unal.edu.co/index.php/revsaludpublica/article/view/44685/62642 
[14] Trottier, H.; Philippe, P. Deterministic Modeling Of Infectious Diseases: Theory And Methods. The Internet Journal of Infectious Diseases, 2000; 1(2):1-6. Recuperado de https://print.ispub.com/api/0/ispub-article/5230

[15] Wainer, G. Discrete Event Modeling and Simulation. A Practitioners Approach. CRC Press, 1st. Edition, 2009; ISBN 9781420053364 - CAT\# 53361. Recuperado de https://www.crcpress.com/ Discrete-Event-Modeling-and-Simulation-A-Practitioners-Approach/Wainer/p/book/ 9781420053364

[16] Wainer, G. $C D++$ : a toolkit to develop DEVS models. Software: Practice and Experience, 2002; 32(13):1261-1306. Recuperado de http://www.sce.carleton.ca/faculty/wainer/papers/spe482.pdf

[17] Wainer, G. Advanced Cell-DEVS modeling applications: a legacy of Norbert Giambiasi. Simulation, 2018; 0(0):1-27. Recuperado de https:// journals.sagepub.com/doi/abs/10.1177/0037549718761596? journal Code= simb

[18] Wainer, G.; Giambiasi, N. Application of the Cell-DEVS paradigm for cell spaces modelling and simulations. Simulation, 2001; 76(1):22-39. Recuperado de https://journals. sagepub.com/doi/10.1177/003754970107600102

[19] Wilchez Velásquez, C. Modelo de propagación de la malaria usando autómatas celulares con indicadores de probabilidad. Undergraduate Thesis, Universidad de los Andes, Bogotá, Colombia, 2014. Recuperado de https://repositorio. uniandes.edu.co/handle/1992/16431 